MATEC Web of Conferences 42, 04007 (2016)

DOI: $10.1051 /$ matecconf/20164204007

(C) Owned by the authors, published by EDP Sciences, 2016

\title{
A Meshless Method for Analysis of 3D Medical Parts
}

\author{
Ming-Hsiao Lee ${ }^{1}$, Jian-Ming Lu ${ }^{1}$ and Keng-Liang $\mathrm{Ou}^{2,3,4,5}$ \\ ${ }^{1}$ National Center for High-performance Computing, Taiwan \\ ${ }^{2}$ School of Dentistry, College of Oral Medicine, Taipei Medical University, Taiwan \\ ${ }^{3}$ Research Center for Biomedical Implants and Microsurgery Devices, Taipei Medical University, Taiwan \\ ${ }^{4}$ Research Center for Biomedical Devices and Prototyping Production, Taipei Medical University, Taiwan \\ ${ }^{5}$ Department of Dentistry, Taipei Medical University-Shuang Ho Hospital, Taiwan
}

\begin{abstract}
A proposed method based on a so-called meshless method is designed to simulate the structural behaviors of 3D medical parts. To do the simulation, the STL (Stereo-Lithography) geometry, which is the main geometry format used in 3D printing and medical models, is adopted to be the analysis model. Moreover, the geometry model of the objects in STL format becomes the only model needed for the simulation. To achieve this, some geometryrelated treatments are required, so some checking processes are designed to handle the analysis model during the analysis. Hence, with the proposed meshless method based on STL geometry, the simulation can be carried out in a simple and straightforward way with less preparation effort for analysis models. A 3D simulation has been conducted to demonstrate the effectiveness and efficiency of this proposed method. Besides, the parts with highly complicated shapes can also be simulated without difficulty.
\end{abstract}

\section{Introduction}

The finite element method (FEM) is a powerful method popularly adopted in dealing with practical engineering problems. However, it causes difficulties during numerical simulations due to some disadvantages. One of the main disadvantages is that it requires element meshes to be the analysis models. To build element meshes is usually a tedious and time-consuming work. Although there are many auto-mesh generators, they are not preferred by the academia and industry because they can only generate tetrahedral elements, instead of favorite hexahedral elements, and randomly create a huge amount of elements, which become difficult to handle for computers and analysts. In addition, the convergence and accuracy of the solutions is strongly affected by the element shape, which often becomes very distorted in large deformation cases. These disadvantages become more serious in dealing with three-dimensional practical engineering problems.

On the contrary, the meshless method, as it is named, does not require any element mesh but only nodes. This method has become one of the most promising numerical methods. Based on similar ideas, there have been emerging various meshless methods, such as, smooth particle hydrodynamics method (SPH) [1], generalized finite difference method [2], diffuse element method [3], particle in cell method [4], element-free Galerkin method (EFGM) [5], partition of unity (PU) [6], the reproducing kernel particle method (RKPM) [7], the $h-p$ clouds [8], the meshless local Petrov-Galerkin method (MLPG) [9], etc.

To analyze part's structural behaviors, the 3D geometry model is needed. Currently, CT-scan and MRI are the useful ways to obtain the geometry data through sliced images in medical field. With the sliced images, 3D models can be formed with triangular facets, which are used to represent the model's surfaces, and then stored in STL (Stereo-Lithography) format, which currently has become a popular geometry format, especially in medical and 3D printing areas.

With the concept of the meshless method and geometry representation in STL format, here proposed is a meshless method based on 'STL geometry'. As mentioned above, the proposed meshless method does not need element meshes. Instead, the STL geometry is adopted. Hence, the geometry model of the objects in STL format becomes the only model needed for the simulation with the proposed method. To achieve this, some geometry-related treatments are required. Herein, some checking processes are proposed and presented to handle those geometry-related situations. Then, with the proposed STL-based meshless method, the simulation becomes simple and straightforward because the STL geometry, which come from CT-scan or MRI, is the only model needed. To demonstrate the effectiveness and efficiency of this proposed method, a 3D medical case has been simulated. Furthermore, even cases with highly irregular geometry, often encountered in medical field, can be easily handled with the proposed method. 


\section{Formulation for meshless method}

The main difference between the FEM and the meshless method is the derivation of the shape functions. In the FEM, the shaped functions are based on the element. In the meshless methods, such as the MLPG, EFGM and most meshless methods, a moving least-squares (MLS) approximation based on surrounding nodes within certain radius is adopted to derive the shape functions [1]. With these shape functions, the numerical formulation for the meshless method can then be carried out.

To implement the weak forms of the governing differential equations numerically, the Galerkin method, as mainly proposed by Belytschko et al. [1], is adopted here. Its procedure is similar to that of the FEM.

\subsection{Moving Least Squares approximation}

As shown in Fig. 1, consider a general three-dimensional linear elastic structure $\Omega$, enclosed by its boundary $\Gamma$.

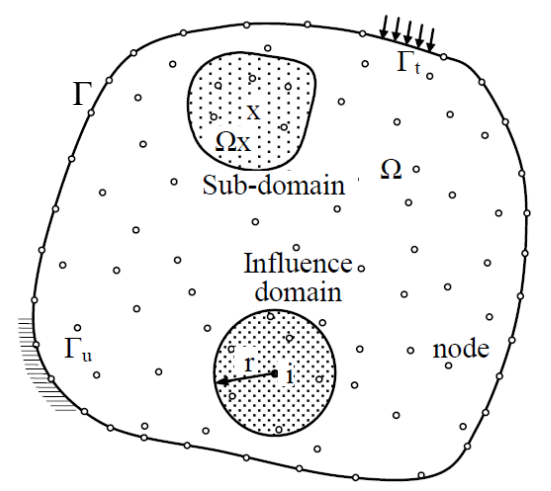

Figure 1. Meshless analysis domain

The unknown field variable $u$ in the sub-domain of the discussed point $\mathrm{x}, \Omega \mathrm{x}$, can be expressed with an approximation function as $u^{h}$,

$u^{h}=\sum_{j=1}^{m} p_{j}(\mathbf{x}) a_{j}(\mathbf{x})=\mathbf{p}^{\mathrm{T}}(\mathbf{x}) \mathbf{a}(\mathbf{x}) \quad$ in $\Omega \mathbf{x}$

where $\mathbf{p}^{\mathrm{T}}(\mathbf{x})=\left[p_{l}(\mathbf{x}), \quad p_{2}(\mathbf{x}), \quad \ldots p_{m}(\mathbf{x})\right]_{1 \times m}$ is a complete monomial basis of order $\mathrm{m}$. $\mathbf{a ( x )}$ are unknown coefficients which are functions of the spatial coordinates $\mathbf{x}$. The coefficients $\mathbf{a}(\mathbf{x})$ can be determined by minimizing a weighted discrete $L_{2}$ norm, which is defined as

$J(\mathbf{x})=\sum_{i=1}^{\mathrm{n}} w_{i}(\mathbf{x})\left[\mathbf{p}^{\mathrm{T}}\left(\mathbf{x}_{i}\right) \mathbf{a}(\mathbf{x})-u_{i}\right]^{2}$

where $w_{i}(\mathbf{x})=w\left(\mathbf{x}-\mathbf{x}_{\mathbf{i}}\right), u_{i}, \mathbf{x}_{i}$ are the weight functions, nodal values, and coordinates associated with node $i$, respectively. $\mathrm{n}$ is the number of nodes in the subdomain. In this work, the following quartic spline function is adopted as the weight function [1]:

$$
w_{i}(\mathbf{x})=\left\{\begin{array}{cc}
1-6\left(\frac{d_{i}}{r_{i}}\right)^{2}+8\left(\frac{d_{i}}{r_{i}}\right)^{3}-3\left(\frac{d_{i}}{r_{i}}\right)^{4} & 0 \leq d_{i} \leq r_{i} \\
0 & d_{i} \geq r_{i}
\end{array}\right.
$$

where $d_{i}=\left|\mathbf{x}-\mathbf{x}_{i}\right|$ denotes the distance between the discussed point and node $i . r_{i}$ is the radius of the influence domain, inside which the things will be influenced by node $i$. The stationary condition of weighted discrete $L_{2}$ norm $J(\mathbf{x})$ with respect to the coefficients $\mathbf{a}(\mathbf{x})$ leads to the relation

$A(x) a(x)=C(x) \hat{u}$

where

$\mathbf{A}(\mathbf{x})=\mathbf{P}^{\mathrm{T}} \mathbf{W} \mathbf{P}$

And

$\mathbf{C}(\mathbf{x})=\mathbf{P}^{\mathrm{T}} \mathbf{W}$

In Eq. (4), $\hat{u}$ is the nodal values and $\mathrm{P}$ is the matrix of the above $\mathrm{pT}(\mathrm{x})$ with the nodal coordinates of those nodes in the subdomain $\Omega \mathrm{x}$, and $\mathrm{W}$ is the diagonal weighting matrix of wi(x). Then, the coefficients $a(x)$ of Eq. (1) can be derived as

$\mathbf{a}(\mathbf{x})=\mathbf{A}(\mathbf{x})^{-1} \mathbf{C}(\mathbf{x}) \hat{\mathbf{u}}$

As in the FEM, $u^{h}$ can also be expressed in the form with shape functions as

$u^{h}=\sum_{j=1}^{n} N_{j}(\mathbf{x}) u_{j}=\mathbf{N}^{\mathrm{T}} \hat{\mathbf{u}}$

Comparing Eq. (8) with Eq. (1), the nodal shape functions $\mathrm{N}$ can thus be derived as

$\mathbf{N}^{\mathbf{T}}(\mathbf{x})=\mathbf{p}^{\mathrm{T}}(\mathbf{x}) \mathbf{A}(\mathbf{x})^{-1} \mathbf{C}(\mathbf{x})$

After the shape functions $\mathbf{N}$ have been derived, the equilibrium equations for analysis problems can then be formed. Based on the principal of minimum total potential energy, the functional $\Pi$ can be represented as

$$
\Pi=\frac{1}{2} \int_{\Omega} \boldsymbol{\varepsilon}^{\mathrm{T}} \mathbf{E} \boldsymbol{\varepsilon} d \Omega-\int_{\Omega} \mathbf{u}^{\mathrm{T}} \cdot \mathbf{b} d \Omega-\int_{\Gamma_{t}} \mathbf{u}^{\mathrm{T}} \cdot \overline{\mathbf{t}} d \Gamma
$$

where $\boldsymbol{\varepsilon}$ is the strain vector, $\mathbf{u}$ is the displacement vector, $\mathbf{b}$ is the body force, $\overline{\mathbf{t}}$ is the prescribed traction applied at the boundary $\Gamma \mathrm{t}$, and $\mathbf{E}$ is the material matrix. The displacement $\mathbf{u}$ at any position of the structure can be interpolated from the neighboring nodal displacement $\mathbf{D}$ by the MLS-based shape function $\boldsymbol{\Psi}$

$\mathbf{u}=\boldsymbol{\Psi} \mathbf{D}$

From the relation between the strain $\varepsilon$ and displacement $\mathbf{u}$, the strain $\varepsilon$ can be expressed as

$$
\boldsymbol{\varepsilon}=\mathbf{B} \mathbf{D}
$$

where $\mathbf{B}$ is the gradient matrix for stains.

Following a similar derivation procedure used in the FEM, the final weak forms of the equilibrium equation can be derived as 
$\mathbf{K} \mathbf{D}=\mathbf{F}$

Where

$$
\mathbf{K}=\int_{\Omega} \mathbf{B}^{\mathrm{T}} \mathbf{E B} d \Omega
$$

and

$$
\mathbf{F}=\int_{\Omega} \boldsymbol{\Psi}^{\mathrm{T}} \mathbf{b} d \Omega+\int_{\Gamma_{t}} \boldsymbol{\Psi}^{\mathrm{T}} \overline{\mathbf{t}} d \Gamma
$$

In the above equations, $\mathbf{K}$ is the global stiffness matrix and $\mathbf{F}$ is the global load vector. After solving the final linear algebraic equations, the field values, i.e. displacements, at any positions of the structure can be obtained.

\section{Geometry-related treatments for the proposed meshless method}

As mentioned above, there are occasions that geometryrelated checking is needed in the meshless analysis, e.g. to determine only those nodes inside the analysis domain to be part of the analysis model.

In order to do that, the STL geometry is adopted to represent three-dimensional analysis domains and all subsequent processes are based on it.

\subsection{STL geometry}

The 3D geometry in STL format, i.e. all surfaces of 3D solid objects are represented by a group of triangular facets, e.g. as shown in Fig 2 is a vertebra model.

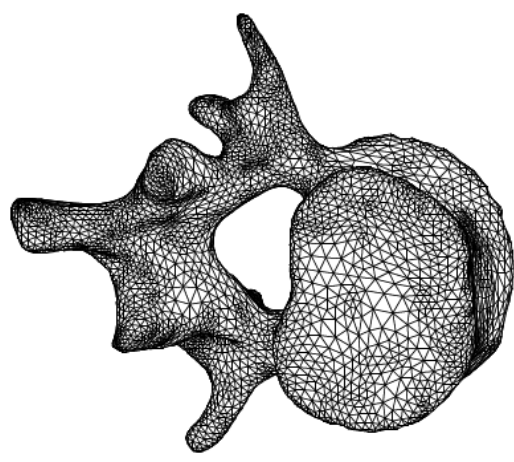

Figure 2. STL geometry of a vertebra

This type of geometry has the flexibility to represent any three-dimensional geometry. It has been widely adopted in many areas currently, such as medical modelling, 3D printing, etc. It is also a powerful way to represent three-dimensional irregular geometry with any free form surfaces. Currently, most computer-aided tools can output the geometry in STL format. In medical field, e.g. through CT-scan or MRI, the medical parts can be represented by STL geometry. Unlike the triangular element used in the FEM, the triangular facet is not the triangular element. Here, the triangular facet is used only to represent geometry.

\subsection{Processes to determine final analysis model}

To determine the final analysis model, as mentioned above, the only geometry data used is a geometry model in STL format. Firstly, import the STL geometry model, as schematically shown in Fig. 3(a). Secondly, pave the nodes to cover the STL geometry model, as shown in Fig. 3(b). The node distribution can be regular or irregular. Basically, regular distribution can be generated automatically by the meshless program with just few programming command lines. Thirdly, exclude those nodes outside the STL geometry model, because some of the regularly generated nodes are outside the analysis domain, as shown in Fig. 3(c). Then, the final analysis model is completed and determined.

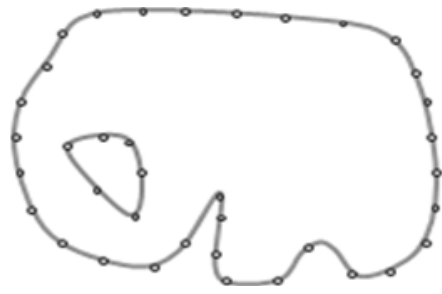

(a) STL geometry (schematically)

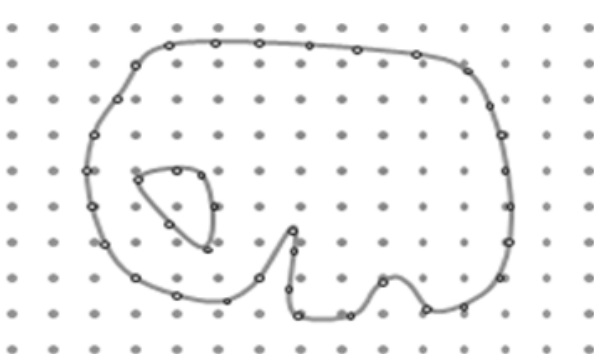

(b) Pave initial nodes

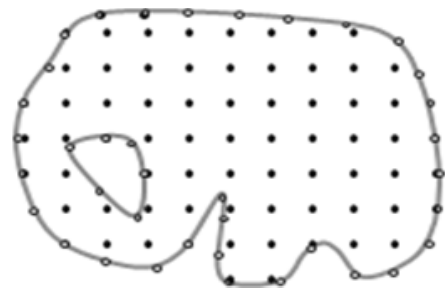

(c) Exclude outside nodes

Fig. 3 Processes to determine the final analysis model

\section{Results and discussion}

To demonstrate the advantages and efficiency of the proposed method, a biomechanics problem is analyzed. In general, the medical parts, either natural or man-made, are used to fit into human's body and their shapes are extremely irregular and complicated. These types of structure always are difficult to deal with. On the contrary, the STL geometry can efficiently represent the complicated medical parts and, with the proposed meshless method, the analysiswork is simple and straightforward that only the STL geometry is needed for doing the analysis. The demonstrative case is a structural 
analysis of a mandible bone without teeth. The mandible bone provides most support during the chewing work. Assume the mandible bone is subjected to a vertical load of $50 \mathrm{~N}$ at the front part, as shown in Fig.4. The Young's modulus of the bone is $10,000 \mathrm{Mpa}$, and the Poisson's ratio is 0.3 . The computed displacement distribution from the proposed method is shown in Fig. 5(a). For comparison, the displacement distribution from the FEM, i.e. ANSYS, is shown in Fig. 5(b). The maximal displacement comparison between these two methods is shown in Table 1. It shows good agreement between the solutions obtained by these two methods, i.e. $0.050 \mathrm{~mm}$ and $0.051 \mathrm{~mm}$, respectively.

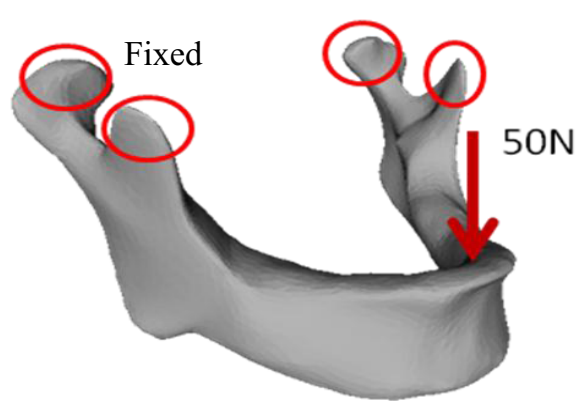

Fig. 4 Geometry model of a mandible

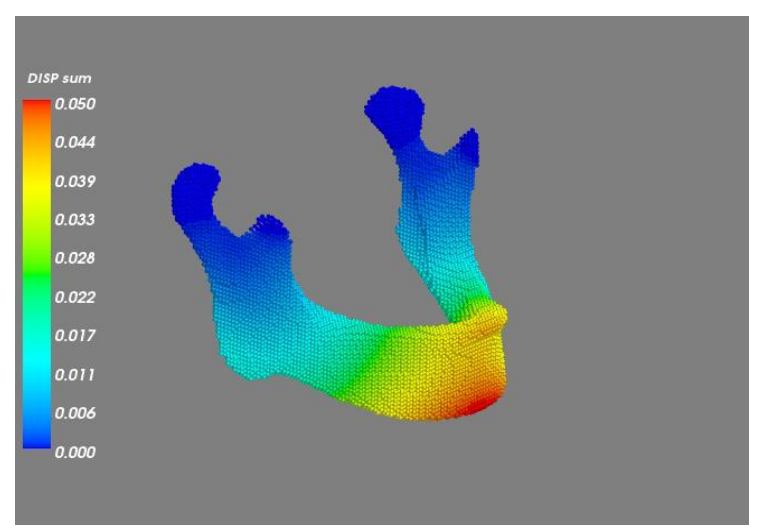

(a) Displacements from proposed method

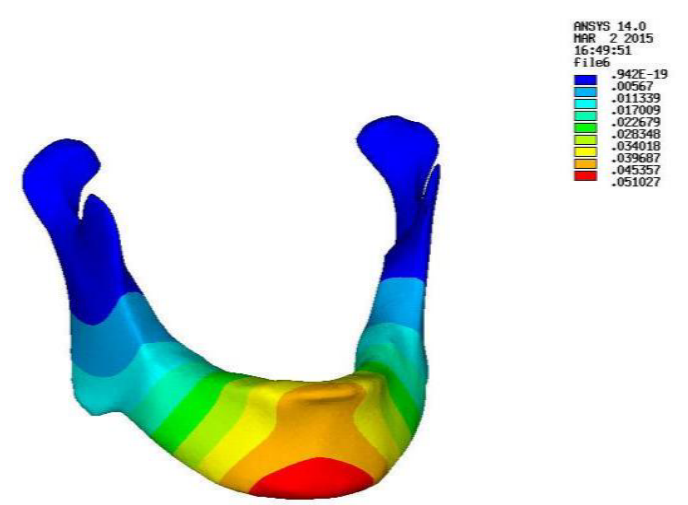

(b) Displacements from ANSYS

Fig. 5 Displacement results from (a) proposed method and (b) ANSYS
Table 1. Max. displacement comparison between the proposed method and ANSYS.

\begin{tabular}{|c|c|}
\hline Method & Max. displacement (mm) \\
\hline Proposed & 0.050 \\
\hline ANSYS & 0.051 \\
\hline
\end{tabular}

\section{Concluding remarks}

The proposed meshless method adopts the STL geometry as the only model for analysis, which can be obtained from CT-scan or MRI, that the analysis model preparation is simple and straightforward. Hence, the tedious and complicated work to prepare the finite element meshes can be avoided. Moreover, the complicated shapes of medical parts can be handled efficiently. Therefore, even for the three-dimensional problems with complicated geometry, the analysis can be conducted without difficulty. Similarly, the parts in STL format in $3 \mathrm{D}$ printing field can also be solved in the same way.

\section{Acknowledgement}

The authors would like to thank the Ministry of Science and Technology of Taiwan for financially supporting this research under Contract MOST-104-2218-E-038-001.

\section{References}

1. R.A. Gingold, J.J. Monaghan, Mon. Not. R. Astron. Soc. 181, 375 - 387 (1977)

2. T. Liszka, J. Orkisz, Comput. Struct. 5, 45 - 58 (1980)

3. B. Nayroles, G. Touzot, P. Villon, Comput. Mech. 10, 307-318. (1992)

4. D. Sulsky, Z. Chen, H.L. Schhreyer, New Methods in Transient Analysis, eds. P. Smolinski, W. K. Liu, G. Hulbert and K. Tamma, AMD-Vol. 143/PVP-Vol. 246, ASME, New York, 91-102 (1992)

5. T. Belytschko, Y.Y. Lu, L. Gu, International Journal for Numerical Methods in Engineering, 37, 229-256 (1994)

6. I. Babuska, J.M. Melenk, Comput. Methods Appl. Mech. Eng. 139, 289-315 (1996)

7. W.K. Liu, S. Jun, Y.F. Zhang, International Journal for Numerical Methods in Fluids, 20, 1081-1106 (1995)

8. C.A. Duarte, J.T. Oden, Comput. Methods Appl. Mech. Engrg., 139, 237-262 (1996)

9. S.N. Atluri, T. Zhu, Comput. Mech., 22, 117-127 (1998) 